\title{
Commentary: Evaluation of the Comorbidity Burden in Patients With Ankylosing Spondylitis Using a Large US Administrative Claims Data Set
}

\author{
Jessica A. Walsh', Xue Song ${ }^{2 *}$, Gilwan Kim², Yujin Park ${ }^{3}$ \\ University of Utah School of Medicine and Salt Lake City Veteran Affairs Medical Center, Division of Rheumatology, Salt Lake City, UT, USA \\ 2IBM Watson Health, Cambridge, MA, USA \\ ${ }^{3}$ Novartis Pharmaceuticals Corporation, East Hanover, NJ, USA
}

Article Info

\section{Article Notes}

Received: October 30, 2018

Accepted: November 30, 2018

\section{*Correspondence:}

Dr. Xue Song, IBM Watson Health, 75 Binney Drive, Cambridge, MA 02142, USA; Telephone No: +1 508.842.5312; Email: songx@us.ibm.com.

(C) 2018 Song $X$. This article is distributed under the terms of the Creative Commons Attribution 4.0 International License.

\section{Keywords}

Chronic inflammatory rheumatic diseases

Ankylosing spondylitis

Comorbidities.

\section{Abstract}

Patients with chronic inflammatory rheumatic diseases, such as rheumatoid arthritis and spondyloarthritis, are at a higher risk of comorbidities, including cardiovascular disease. Although the prevalence of spondyloarthritis is estimated to be similar to that of rheumatoid arthritis, the risk of cardiovascular comorbidities in spondyloarthritis is not as well understood. Furthermore, the inflammatory rheumatic diseases differ in their pathogenic mechanisms, the populations affected, and treatment recommendations; therefore, it is important to examine these diseases separately. Ankylosing spondylitis (AS) is the prototype of spondyloarthritis; the onset of disease occurs at a relatively young age, and patients with AS are often undiagnosed for long periods of time. This increased duration of exposure to inflammation and use of nonsteroidal anti-inflammatory drugs may contribute to the higher risk of cardiovascular comorbidities in these patients.

Here we describe our recently published study (Walsh JA, et al. Clin Rheumatol. 2018;37[7]:1869-1878.), which used large national claims databases and showed that US patients with AS had significantly more comorbidities, including cardiovascular disease, than matched controls. We also review the current understanding of the risk of cardiovascular comorbidities in patients with AS. Knowledge of the frequency and risk of comorbidities can assist rheumatologists and primary care physicians with comorbidity screening and strategies for a holistic care approach for patients with AS, including the possibility of adapting the existing cardiovascular risk assessments for these patients. Counseling patients on additional lifestyle risk factors, early cardiovascular screening, and the necessity of further diagnostic testing will be important for optimizing patient care for AS.

\section{Cardiovascular Disease in Patients With Inflammatory Rheumatic Diseases}

Patients with chronic inflammatory rheumatic diseases, such as rheumatic arthritis, spondyloarthritis, and systemic lupus erythematosus, have an increased risk of cardiovascular disease ${ }^{1-8}$. The increased cardiovascular risk in patients with inflammatory rheumatic disease is likely related to systemic inflammation and traditional cardiovascular risk factors, such as hypertension, dyslipidemia, diabetes, smoking, and obesity, some of which are more prevalent in patients with rheumatic diseases. A link between inflammation and accelerated atherosclerosis has been identified in patients with inflammatory rheumatic disease ${ }^{9-11}$. Furthermore, endothelial dysfunction, oxidative stress, macrophage accumulation, toll-like receptor signaling, and proinflammatory cytokines have been implicated in atherogenesis ${ }^{9,11,12}$. Similar to the heterogeneity in 
traditional cardiovascular risk factors, there are differences between the autoimmune and inflammatory risk factors of rheumatic diseases; therefore, cardiovascular risk assessment and treatment should be tailored for each rheumatic disease. In addition to traditional risk factors and systemic inflammation, the use of specific nonsteroidal anti-inflammatory drugs (NSAIDs), which are often used in the management of some inflammatory rheumatic diseases, may play a role in the risk of cardiovascular disease ${ }^{13-16}$.

The Prototype of Spondyloarthritis: Ankylosing Spondylitis

Spondyloarthritis represents a group of inflammatory rheumatic disorders comprising ankylosing spondylitis (AS), nonradiographic axial spondyloarthritis, psoriatic arthritis, reactive arthritis, arthritis associated with inflammatory bowel disease, and undifferentiated spondyloarthropathies. With estimates of an overall prevalence of $>1 \%$ in the United States ${ }^{17}$, spondyloarthritis is at least as common as rheumatoid arthritis among whites ${ }^{18-21}$ and is one of the most common chronic inflammatory disorders. Spondyloarthritis is characterized by peripheral arthritis and enthesitis, axial inflammation (ie, sacroiliitis and spondylitis), and new bone formation leading to ankylosis. Because spondyloarthritis develops relatively early in life and has a chronic, progressive course, the impact of the disease on patients can be substantial.

Prevalence of AS in the United States has been estimated between $0.2 \%$ and $0.5 \% 17,22-24$. Although the age of onset is typically the late teens through 40 years of age, delays in diagnosis by as much as 8 to 11 years may lead to diagnoses at an older age $\mathrm{e}^{25-27}$. In addition to inflammation of the spine, joints, and entheses, patients with AS often present with peripheral arthritis, uveitis, psoriasis, and inflammatory bowel diseases. Furthermore, studies have shown that compared with the general population, patients with AS are at a higher risk of developing comorbidities including cardiovascular disease, diabetes, malignancies, and depression ${ }^{6,14,28-38}$.

Although previous studies of comorbidities in patients with AS have provided important information, most of these studies have been conducted outside of the United States. Because rates of comorbidities in the general population differ between the United States and other countries, there is a need to further understand comorbidities in US patients with AS. Here, we discuss the results of a recent real-world study, which examined the comorbidity burden of US patients with AS using a large national healthcare claims database. In addition, we review the current understanding of the risk of cardiovascular comorbidities in patients with AS.

\section{Comorbidities in AS}

Our recently published real-world study (Walsh JA, et al. Clin Rheumatol. 2018;37[7]:1869-1878.) compared the prevalence and incidence of comorbidities between patients with AS and matched controls using medical and pharmacy claims data from the MarketScan ${ }^{\circledR}$ Commercial and Medicare databases from 2012 through 2015. A total of 6679 patients with medical claims for AS were matched with 19,951 patients without AS at a ratio of up to 1:5 based on age, geographic location, index calendar year, and $\operatorname{sex}^{39}$. Patients with AS had a mean (SD) age of 50.8 (13.6) years, and $60.5 \%$ were men; matched controls had a mean age of 51.7 (13.4) years, and $60.8 \%$ were men ${ }^{39}$. The mean (SD) length of follow-up in patients with AS and in matched controls was 739 (139) days and 740 (139) days, respectively ${ }^{39}$. Patients with AS had a higher baseline comorbidity burden than matched controls (mean [SD] Deyo-Charlson Comorbidity Index score, 0.61 [1.15] vs 0.50 [1.14], $P<0.001$ ) and were significantly more likely to have diagnoses of asthma, cardiovascular diseases, depression, dyslipidemia, gastrointestinal ulcers, malignancies, multiple sclerosis, osteoporosis, sleep apnea, spinal fracture, inflammatory bowel diseases, psoriasis, and uveitis (Table 1$)^{39}$.

Patients with AS had significantly higher incidence rates of all other comorbidities compared with matched controls, except for diabetes, dyslipidemia, and Parkinson disease

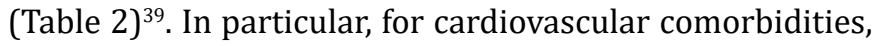
patients with AS had an approximately 1.25× higher incidence rate of angina, atherosclerosis, cerebrovascular disease/stroke, coronary artery disease, hypertension, myocardial infarction, and peripheral vascular disease and $2 \times$ higher incidence of venous thromboembolism compared with matched controls (Figure 1$)^{39}$. The risk for cardiovascular disease persisted after statistical adjustments for baseline characteristics and comorbidities (including hypertension), as demonstrated in the published manuscript ${ }^{39}$. An important limitation of our study was the lack of body mass index data; therefore, obesity could not be evaluated as a comorbidity or be controlled for with related comorbidities such as cardiovascular disease and diabetes $^{39}$. In addition, other risk factors that could have contributed to the development of comorbidities (eg, family history, smoking, alcohol consumption, and the use of over-the-counter NSAIDs) were not available in the data $\operatorname{set}^{39}$.

Although our study did not examine the causality of cardiovascular comorbidities in patients with AS, the chronic inflammatory state of the disease may be linked to the development of these comorbidities ${ }^{40}$, as seen in rheumatoid arthritis ${ }^{41}$. In addition, onset of disease begins 
Table 1: Baseline clinical characteristics and comorbidities in patients with AS and their matched controls

\begin{tabular}{|c|c|c|c|}
\hline Comorbidities & $\begin{array}{c}\text { Patients } \\
\text { With AS } \\
(n=6679)\end{array}$ & $\begin{array}{l}\text { Matched Controls } \\
\quad(n=19,951)\end{array}$ & $P$ Value \\
\hline Deyo-Charlson Comorbidity Index, mean (SD) & $0.61(1.15)$ & $0.50(1.14)$ & $<0.001$ \\
\hline \multicolumn{4}{|l|}{ Comorbidities, $\mathbf{n}(\%)$} \\
\hline Asthma & $145(2.2)$ & $218(1.1)$ & $<0.001$ \\
\hline Cardiovascular disease & $2295(34.4)$ & $5849(29.3)$ & $<0.001$ \\
\hline Angina & $79(1.2)$ & $146(0.7)$ & $<0.001$ \\
\hline Atherosclerosis & $482(7.2)$ & $1139(5.7)$ & $<0.001$ \\
\hline Cerebrovascular disease/stroke & $110(1.6)$ & $260(1.3)$ & 0.038 \\
\hline Coronary artery disease & $322(4.8)$ & $743(3.7)$ & $<0.001$ \\
\hline Hypertension & $2053(30.7)$ & $5267(26.4)$ & $<0.001$ \\
\hline Myocardial infarction & $84(1.3)$ & $177(0.9)$ & 0.009 \\
\hline Peripheral vascular disease & $110(1.6)$ & $311(1.6)$ & 0.617 \\
\hline Venous thromboembolism & $77(1.2)$ & $167(0.8)$ & 0.019 \\
\hline Depression & $712(10.7)$ & $1152(5.8)$ & $<0.001$ \\
\hline Diabetes & $656(9.8)$ & $2113(10.6)$ & 0.075 \\
\hline Dyslipidemia & $1564(23.4)$ & $4439(22.3)$ & 0.048 \\
\hline Gastrointestinal ulcers & $75(1.1)$ & $79(0.4)$ & $<0.001$ \\
\hline Malignancies & $450(6.7)$ & $1094(5.5)$ & $<0.001$ \\
\hline Multiple sclerosis & $31(0.5)$ & $51(0.3)$ & 0.008 \\
\hline Osteoporosis & 259 (3.9) & $191(1.0)$ & $<0.001$ \\
\hline Parkinson disease & $17(0.3)$ & $43(0.2)$ & 0.561 \\
\hline Sleep apnea & $585(8.8)$ & $1008(5.1)$ & $<0.001$ \\
\hline Spinal fracture & $59(0.9)$ & $39(0.2)$ & $<0.001$ \\
\hline \multicolumn{4}{|l|}{ Extra-articular manifestations of AS, \% } \\
\hline Inflammatory bowel disease & $439(6.6)$ & $128(0.6)$ & $<0.001$ \\
\hline Crohn disease & $281(4.2)$ & $57(0.3)$ & $<0.001$ \\
\hline Ulcerative colitis & $192(2.9)$ & $81(0.4)$ & $<0.001$ \\
\hline Psoriasis & $138(2.1)$ & $171(0.9)$ & $<0.001$ \\
\hline Uveitis & $654(9.8)$ & $45(0.2)$ & $<0.001$ \\
\hline
\end{tabular}

AS, ankylosing spondylitis.

a Deyo-Charlson Comorbidity Index ranges from 0 to 33.

Source: Walsh JA, et al. Clin Rheumatol. 2018; 37(7): 1869-1878. (https://creativecommons.org/licenses/by/4.0/)

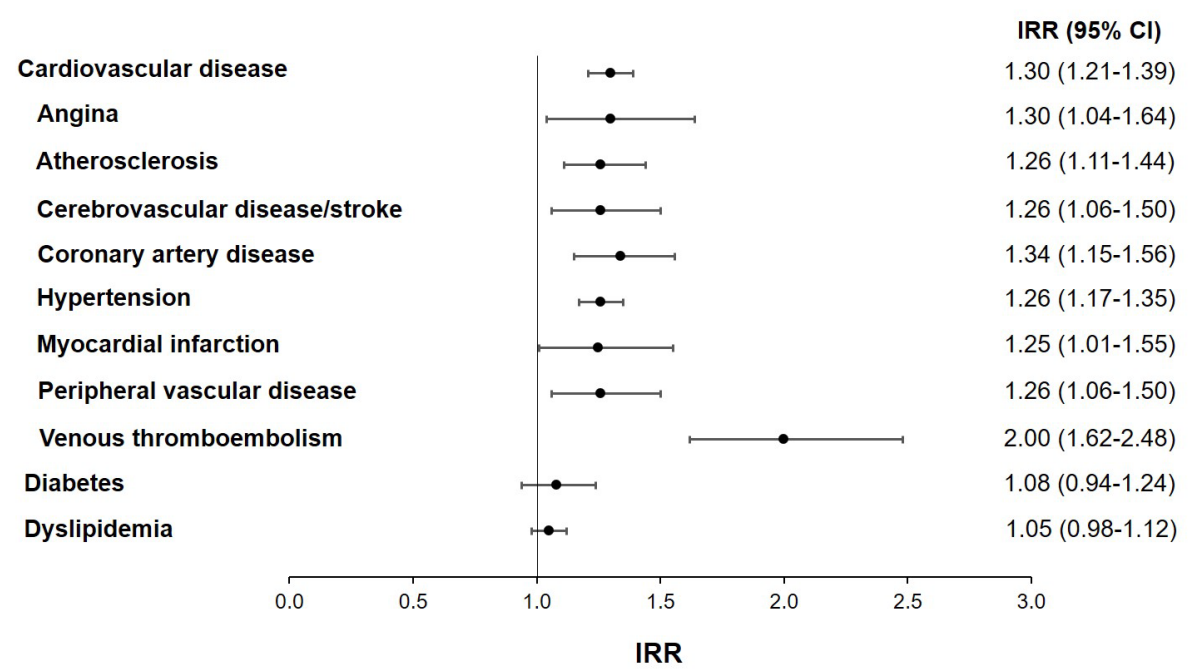

Figure 1: Incidence rate ratios of comorbidities in patients with ankylosing spondylitis vs their matched controls. IRR, incidence rate ratio. 
Table 2: Proportions of patients with new comorbidities and the incidence rates per 100 patient-years

\begin{tabular}{|c|c|c|c|c|c|}
\hline & \multicolumn{2}{|c|}{$\begin{array}{l}\text { Patients With AS } \\
\qquad N=6679\end{array}$} & \multicolumn{2}{|c|}{$\begin{array}{l}\text { Matched Controls } \\
\qquad N=19,951\end{array}$} & \multirow{2}{*}{$P$ Value } \\
\hline & n (\%) & $\begin{array}{l}\text { Incidence } \\
\text { rate }\end{array}$ & n (\%) & $\begin{array}{l}\text { Incidence } \\
\text { rate }\end{array}$ & \\
\hline \multicolumn{6}{|l|}{ Comorbidities } \\
\hline Asthma & $178(2.7)$ & 1.37 & $302(1.5)$ & 0.76 & $<0.001$ \\
\hline Cardiovascular disease & $1080(16.2)$ & 14.36 & $2795(14.0)$ & 11.06 & $<0.001$ \\
\hline Angina & $104(1.6)$ & 0.79 & $240(1.2)$ & 0.60 & 0.027 \\
\hline Atherosclerosis & $328(4.9)$ & 2.69 & $794(4.0)$ & 2.13 & 0.001 \\
\hline Cerebrovascular disease/stroke & $185(2.8)$ & 1.41 & $442(2.2)$ & 1.12 & 0.010 \\
\hline Coronary artery disease & $233(3.5)$ & 1.85 & $529(2.7)$ & 1.38 & $<0.001$ \\
\hline Hypertension & $1044(15.6)$ & 12.98 & $2736(13.7)$ & 10.33 & $<0.001$ \\
\hline Myocardial infarction & $118(1.8)$ & 0.89 & $284(1.4)$ & 0.71 & 0.046 \\
\hline Peripheral vascular disease & $177(2.70$ & 1.35 & $422(2.1)$ & 1.07 & 0.011 \\
\hline Venous thromboembolism & $142(2.1)$ & 1.07 & $214(1.1)$ & 0.54 & $<0.001$ \\
\hline Depression & $686(10.3)$ & 6.05 & $1187(6.0)$ & 3.22 & $<0.001$ \\
\hline Diabetes & $273(4.1)$ & 2.30 & $752(3.8)$ & 2.13 & 0.242 \\
\hline Dyslipidemia & $1208(18.1)$ & 13.52 & $3544(17.8)$ & 12.90 & 0.551 \\
\hline Gastrointestinal ulcers & $93(1.4)$ & 0.70 & $156(0.8)$ & 0.39 & $<0.001$ \\
\hline Malignancies & $394(5.9)$ & 3.24 & $879(4.4)$ & 2.35 & $<0.001$ \\
\hline Multiple sclerosis & $19(0.3)$ & 0.14 & $16(0.1)$ & 0.04 & $<0.001$ \\
\hline Osteoporosis & $286(4.3)$ & 2.26 & $250(1.3)$ & 0.63 & $<0.001$ \\
\hline Parkinson disease & $14(0.2)$ & 0.10 & $32(0.2)$ & 0.08 & 0.402 \\
\hline Sleep apnea & $445(6.7)$ & 3.76 & $827(4.1)$ & 2.21 & $<0.001$ \\
\hline Spinal fracture & $107(1.6)$ & 0.81 & $89(0.4)$ & 0.22 & $<0.001$ \\
\hline \multicolumn{6}{|l|}{ Extra-articular manifestations of AS } \\
\hline Inflammatory bowel disease & $209(3.1)$ & 1.69 & $85(0.4)$ & 0.21 & $<0.001$ \\
\hline Crohn disease & $127(1.9)$ & 0.99 & $40(0.2)$ & 0.10 & $<0.001$ \\
\hline Ulcerative colitis & $136(2.0)$ & 1.05 & $59(0.3)$ & 0.15 & $<0.001$ \\
\hline Psoriasis & $202(3.0)$ & 1.55 & $180(0.9)$ & 0.45 & $<0.001$ \\
\hline Uveitis & $469(7.0)$ & 4.04 & $60(0.3)$ & 0.15 & $<0.001$ \\
\hline
\end{tabular}

AS, ankylosing spondylitis.

Source: Walsh JA, et al. Clin Rheumatol. 2018; 37(7): 1869-1878. (https://creativecommons.org/licenses/by/4.0/)

earlier in patients with AS compared with rheumatoid arthritis, and patients with AS are often undiagnosed for longer periods of time without having their underlying inflammation managed ${ }^{25-27}$; therefore, the increased duration of uncontrolled inflammation may contribute to the higher risk of cardiovascular comorbidities in patients with AS. Furthermore, in patients with AS, NSAIDs are recommended as first-line therapy ${ }^{42-45}$ and may be used more commonly and persistently in patients with AS than in those with other inflammatory rheumatic diseases. Further research is needed to evaluate the potential cause and effect relationships between AS and comorbidities.

The elevated risk of cardiovascular disease in patients with AS shown in our study ${ }^{39}$ supported evidence from published reports on the risk of developing new cardiovascular comorbidities in patients with $\mathrm{AS}^{14,35,46}$. A study from the Swedish National Patient Register showed a $50 \%$ higher risk of acute coronary syndrome and vascular thromboembolism and a $25 \%$ higher risk of stroke in patients with AS compared with the general population ${ }^{46}$.
A meta-analysis of 18 studies of patients with AS and 12 studies of control patients reported a relative risk of myocardial infarction of 1.44 (95\% CI, 1.25-1.67) in patients with AS compared with controls ${ }^{6}$. The same study also reported the results of a meta-analysis of 7 studies and reported a relative risk of stroke of 1.37 (95\% CI, 1.08$1.73)^{6}$. Furthermore, an administrative claims study from the Taiwan National Health Insurance Database showed a $>2$-fold increase in the risk of stroke in patients with AS compared with a comparison cohort without $\mathrm{AS}^{35}$. Notably, the increased risk of cardiovascular disease in patients with AS was demonstrated globally despite geographic differences in baseline cardiovascular disease risk in the general population.

Not all cardiac outcomes were assessed in our study. Valvular heart disease and conduction abnormalities are of interest in AS because they have been linked to aortitis and HLA-B27 positivity. In a study of Medicare beneficiaries over the age of 65 years, statistically higher risks were reported for mitral and aortic valve disease (OR, 1.06-1.51) in AS patients 
$(\mathrm{n}=42,327)$ vs controls $(\mathrm{n}=19,211,703)^{47}$. Rates of aortic valve procedures were also statistically higher in AS patients than controls (OR, 1.22-1.46), but rates of mitral valve procedures were similar between groups ${ }^{47}$. In addition, pacemaker insertions were evaluated as an estimate of serious and symptomatic conduction abnormalities and were more frequent in patients with AS than controls (OR, 1.11-1.32), particularly in older age groups ${ }^{47}$. These small risk differences do not support routine screening for valvular heart disease or conduction abnormalities in asymptomatic AS patients.

The European League Against Rheumatism (EULAR) recommendations for cardiovascular disease risk management advise clinicians to be aware of the higher risk of cardiovascular disease in patients with inflammatory joint disease and screen patients for cardiovascular risk at least every 5 years and following changes in antirheumatic therapy ${ }^{1}$. Commonly used cardiovascular risk assessments in the general population are the Framingham Risk Score, the Systematic Coronary Risk Evaluation (SCORE), the Reynolds Risk Score, and the QRESEARCH Cardiovascular Risk Algorithm (QRisk) 2 score. However, these risk assessments may underestimate the cardiovascular disease risk in patients with AS because nontraditional cardiovascular risk factors are not included. The use of a relative risk chart has also been proposed as an alternative to the Systematic Coronary Risk Evaluation in patients aged $<50$ years to determine the risk of cardiovascular disease ${ }^{48}$. Furthermore, the European League Against Rheumatism recommendations advise adapting cardiovascular risk assessments for patients with rheumatoid arthritis with a multiplication factor of $1.5^{1}$. Whether this multiplication factor should also apply to patients with AS remains unclear, but it may be an appropriate option in the absence of risk prediction models with proven accuracy and superiority in patients with inflammatory joint disease.

Screening for asymptomatic atherosclerotic plaques using carotid ultrasound is recommended in patients with rheumatoid arthrits ${ }^{1}$ and may be appropriate for patients with AS, especially younger patients ${ }^{48}$. Patients with AS are generally younger than patients with rheumatoid arthritis, and as a result, they may not receive the same cardiovascular screening. Because of the increased risk of cardiovascular disease in patients with AS compared with the general population, monitoring for cardiovascular disease may be needed at an earlier age than what is traditionally recommended for patients without AS.

Prompt recognition and treatment of cardiovascular risk factors is important to decrease the morbidity and mortality associated with cardiovascular disease. Furthermore, the age and demographic characteristics of the individual patient must be considered. Patients with AS are diagnosed at a younger age than those with rheumatoid arthritis and are more likely to be male, which also increases their risk of cardiovascular disease. How age affects cardiovascular disease risk in patients with AS is unknown, although it has been explored in other inflammatory rheumatic diseases. Notably, younger women with systemic lupus erythematosus have a higher relative risk of cardiovascular disease compared with the general population than women with systemic lupus erythematosus who are $>60$ years of age ${ }^{7}$.

\section{Conclusions}

Our AS comorbidities study ${ }^{39}$, which evaluated a large real-world sample of patients with AS, was among the first to evaluate comorbidities, including cardiovascular comorbidities, in US patients with AS compared with matched controls. Our study provides important information about the increased risks of comorbidities in US patients with AS, and research is needed to evaluate potential relationships between inflammation and comorbidities in patients with AS.

Knowledge of the frequency and risk of comorbidities can assist rheumatologists and primary care physicians with comorbidity screening and strategies for management in patients with AS. Importantly, in addition to lifestyle management and counseling related to the traditional risk factors of cardiovascular disease, patients with AS may need diagnostic screening for cardiovascular disease at an earlier age than patients without AS, as well as further modification of the standard cardiovascular risk assessments. Furthermore, tailoring recommendations and treatment based on studies in patients with AS instead of adapting existing recommendations based on studies in patients with other inflammatory rheumatic diseases may provide optimal care for patients with AS.

\section{Funding}

This commentary was sponsored by Novartis Pharmaceuticals Corporation, East Hanover, NJ, USA.

\section{Authors' Full Disclosures}

Jessica Walsh is a paid consultant to Novartis. Xue Song and Gilwan Kim are employees of IBM Watson Health, which was paid by Novartis in connection with the development of this manuscript. Yujin Park is an employee of Novartis.

\section{Acknowledgements}

Support for third-party writing assistance, furnished by Nicola Gillespie, DVM, of Health Interactions, Inc, was provided by Novartis Pharmaceuticals Corporation, East Hanover, NJ.

\section{References}

1. Agca R, Heslinga SC, Rollefstad S, et al. EULAR recommendations for cardiovascular disease risk management in patients with rheumatoid arthritis and other forms of inflammatory joint disorders: 2015/2016 update. Ann Rheum Dis. 2017; 76(1): 17-28. 
2. Horreau C, Pouplard C, Brenaut E, et al. Cardiovascular morbidity and mortality in psoriasis and psoriatic arthritis: A systematic literature review. J Eur Acad Dermatol Venereol. 2013; 27 Suppl 3: 12-29.

3. Hu SC, Lan CE. Psoriasis and cardiovascular comorbidities: Focusing on severe vascular events, cardiovascular risk factors and implications for treatment. Int J Mol Sci. 2017; 18(10): 10.3390/ijms18102211.

4. Avina-Zubieta JA, Choi HK, Sadatsafavi M, et al. Risk of cardiovascular mortality in patients with rheumatoid arthritis: A meta-analysis of observational studies. Arthritis Rheum. 2008; 59(12): 1690-1697.

5. Gabriel SE. Cardiovascular morbidity and mortality in rheumatoid arthritis. Am J Med. 2008; 121(10 Suppl 1): S9-14.

6. Mathieu S, Soubrier M. Cardiovascular events in ankylosing spondylitis: A 2018 meta-analysis. Ann Rheum Dis. 2018.

7. Schoenfeld SR, Kasturi S, Costenbader KH. The epidemiology of atherosclerotic cardiovascular disease among patients with SLE: A systematic review. Semin Arthritis Rheum. 2013; 43(1): 77-95.

8. Han C, Robinson DWJ, Hackett MV, et al. Cardiovascular disease and risk factors in patients with rheumatoid arthritis, psoriatic arthritis, and ankylosing spondylitis. J Rheumatol. 2006; 33(11): 2167-2172.

9. Murdaca G, Colombo BM, Cagnati P, et al. Endothelial dysfunction in rheumatic autoimmune diseases. Atherosclerosis. 2012; 224(2): 309317.

10. Nurmohamed MT, Heslinga M, Kitas GD. Cardiovascular comorbidity in rheumatic diseases. Nat Rev Rheumatol. 2015; 11(12): 693-704.

11. Steyers CM 3rd, Miller FJ Jr. Endothelial dysfunction in chronic inflammatory diseases. Int J Mol Sci. 2014; 15(7): 11324-11349.

12. Arida A, Protogerou AD, Kitas GD, et al. Systemic inflammatory response and atherosclerosis: The paradigm of chronic inflammatory rheumatic diseases. Int J Mol Sci. 2018;19(7):10.3390/ijms19071890.

13. Dubreuil M, Louie-Gao Q Peloquin CE, et al. Risk of myocardial infarction with use of selected non-steroidal anti-inflammatory drugs in patients with spondyloarthritis and osteoarthritis. Ann Rheum Dis. 2018; 77(8): 1137-1142.

14. Essers I, Stolwijk C, Boonen A, et al. Ankylosing spondylitis and risk of ischaemic heart disease: A population-based cohort study. Ann Rheum Dis. 2016; 75(1): 203-209.

15. Roubille C, Richer V, Starnino T, et al. The effects of tumour necrosis factor inhibitors, methotrexate, non-steroidal anti-inflammatory drugs and corticosteroids on cardiovascular events in rheumatoid arthritis, psoriasis and psoriatic arthritis: A systematic review and meta-analysis. Ann Rheum Dis. 2015; 74(3): 480-489.

16. Lindhardsen J, Gislason GH, Jacobsen S, et al. Non-steroidal antiinflammatory drugs and risk of cardiovascular disease in patients with rheumatoid arthritis: A nationwide cohort study. Ann Rheum Dis. 2014; 73(8): 1515-1521.

17. Reveille JD. Epidemiology of spondyloarthritis in North America. Am J Med Sci. 2011; 341(4): 284-286.

18. Hunter TM, Boytsov NN, Zhang X, et al. Prevalence of rheumatoid arthritis in the United States adult population in healthcare claims databases, 2004-2014. Rheumatol Int. 2017; 37(9): 1551-1557.

19. Helmick CG, Felson DT, Lawrence RC, et al. Estimates of the prevalence of arthritis and other rheumatic conditions in the United States. Part I. Arthritis Rheum. 2008; 58(1): 15-25.

20. Alamanos Y, Voulgari PV, Drosos AA. Incidence and prevalence of rheumatoid arthritis, based on the 1987 American College of Rheumatology criteria: A systematic review. Semin Arthritis Rheum. 2006; 36(3): 182-188

21. Crane MM, Juneja M, Allen J, et al. Epidemiology and treatment of new-onset and established rheumatoid arthritis in an insured US population. Arthritis Care Res (Hoboken). 2015; 67(12): 1646-1655.
22. Curtis JR, Harrold LR, Asgari MM, et al. Diagnostic prevalence of ankylosing spondylitis using computerized health care data, 1996 to 2009: Underrecognition in a US health care setting. Perm J. 2016; 20(4): 4-10.

23. Strand V, Rao SA, Shillington AC, et al. Prevalence of axial spondyloarthritis in United States rheumatology practices: Assessment of SpondyloArthritis International Society criteria versus rheumatology expert clinical diagnosis. Arthritis Care Res (Hoboken). 2013; 65(8): 1299-1306

24. Reveille JD, Weisman MH. The epidemiology of back pain, axial spondyloarthritis and HLA-B27 in the United States. Am J Med Sci. 2013; 345(6): 431-436.

25. Braun J, Sieper J. Ankylosing spondylitis. Lancet. 2007; 369(9570): $1379-1390$

26. Feldtkeller E, Khan MA, van der Heijde D, et al. Age at disease onset and diagnosis delay in HLA-B27 negative vs. positive patients with ankylosing spondylitis. Rheumatol Int. 2003; 23(2): 61-66.

27. Deodhar A, Mease PJ, Reveille JD, et al. Frequency of axial spondyloarthritis diagnosis among patients seen by United States rheumatologists for evaluation of chronic back pain. Arthritis Rheumatol. 2016; 68(7): 1669-1676.

28. Kang JH, Chen YH, Lin HC. Comorbidity profiles among patients with ankylosing spondylitis: A nationwide population-based study. Ann Rheum Dis. 2010; 69(6): 1165-1168.

29. Bremander A, Petersson IF, Bergman S, et al. Population-based estimates of common comorbidities and cardiovascular disease in ankylosing spondylitis. Arthritis Care Res (Hoboken). 2011; 63(4): 550-556.

30. Mercieca C, van der Horst-Bruinsma IE, Borg AA. Pulmonary, renal and neurological comorbidities in patients with ankylosing spondylitis; implications for clinical practice. Curr Rheumatol Rep. 2014; 16(8): 434-014-0434-7.

31. Chou CH, Lin MC, Peng CL, et al. A nationwide population-based retrospective cohort study: Increased risk of acute coronary syndrome in patients with ankylosing spondylitis. Scand J Rheumatol. 2014; 43(2): 132-136.

32. Meesters JJ, Bremander A, Bergman S, et al. The risk for depression in patients with ankylosing spondylitis: A population-based cohort study. Arthritis Res Ther. 2014; 16(5): 418-014-0418-z.

33. Sun LM, Muo CH, Liang JA, et al. Increased risk of cancer for patients with ankylosing spondylitis: A nationwide population-based retrospective cohort study. Scand J Rheumatol. 2014; 43(4): 301-306.

34. Chen HH, Yeh SY, Chen HY, et al. Ankylosing spondylitis and other inflammatory spondyloarthritis increase the risk of developing type 2 diabetes in an Asian population. Rheumatol Int. 2014; 34(2): 265-270.

35. Keller JJ, Hsu JL, Lin SM, et al. Increased risk of stroke among patients with ankylosing spondylitis: A population-based matched-cohort study. Rheumatol Int. 2014; 34(2): 255-263.

36. Rosenbaum J, Chandran V. Management of comorbidities in ankylosing spondylitis. Am J Med Sci. 2012; 343(5): 364-366.

37. Castaneda S, Martin-Martinez MA, Gonzalez-Juanatey C, et al Cardiovascular morbidity and associated risk factors in Spanish patients with chronic inflammatory rheumatic diseases attending rheumatology clinics: Baseline data of the CARMA project. Semin Arthritis Rheum. 2015; 44(6): 618-626.

38. Bergman M, Lundholm A. Managing morbidity and treatment-related toxicity in patients with ankylosing spondylitis. Rheumatology (Oxford). 2018; 57(3): 419-428.

39. Walsh JA, Song X, Kim G, et al. Evaluation of the comorbidity burden in patients With Ankylosing Spondylitis Using a Large US Administrative Claims Data Set. Clin Rheumatol. 2018; 37(7): 1869-1878. 
40. Lauper K, Courvoisier DS, Chevallier P, et al. Incidence and prevalence of major adverse cardiovascular events in rheumatoid arthritis, psoriatic arthritis, and axial spondyloarthritis. Arthritis Care Res (Hoboken). 2018.

41. Zegkos T, Kitas G, Dimitroulas T. Cardiovascular risk in rheumatoid arthritis: Assessment, management and next steps. Ther Adv Musculoskelet Dis. 2016; 8(3): 86-101.

42. van der Heijde D, Ramiro S, Landewe R, et al. 2016 update of the ASASEULAR management recommendations for axial spondyloarthritis. Ann Rheum Dis. 2017; 76(6): 978-991.

43. Ward MM, Deodhar A,Akl EA, et al. American College of Rheumatology/ Spondylitis Association of America/Spondyloarthritis Research and Treatment Network 2015 recommendations for the treatment of ankylosing spondylitis and nonradiographic axial spondyloarthritis. Arthritis Rheumatol. 2016; 68(2): 282-298.

44. Kroon FP, van der Burg LR, Ramiro S, et al. Non-steroidal antiinflammatory drugs (NSAIDs) for axial spondyloarthritis (ankylosing spondylitis and non-radiographic axial spondyloarthritis). Cochrane Database Syst Rev. 2015; 7: CD010952.

45. Wanders A, Heijde D, Landewe R, et al. Nonsteroidal antiinflammatory drugs reduce radiographic progression in patients with ankylosing spondylitis: A randomized clinical trial. Arthritis Rheum. 2005; 52(6): 1756-1765.

46. Bengtsson K, Forsblad-d'Elia H, Lie E, et al. Are ankylosing spondylitis, psoriatic arthritis and undifferentiated spondyloarthritis associated with an increased risk of cardiovascular events? A prospective nationwide population-based cohort study. Arthritis Res Ther. 2017; 19(1): 102-017-1315-z.

47. Ward MM. Lifetime risks of valvular heart disease and pacemaker use in patients with ankylosing spondylitis. J Am Heart Assoc. 2018; 7(20): e010016.

48. Rueda-Gotor J, Genre F, Corrales A, et al. Relative risk chart score for the assessment of the cardiovascular risk in young patients with ankylosing spondylitis. Int J Rheumatol. 2018; 2018: 1847894. 